\title{
Necessary and sufficient Tauberian conditions for weighted mean methods of summability in two-normed spaces
}

\author{
İbrahim Çanak, Gizem Erikli, Sefa Anil Sezer, and Ece Yaraşgil
}

\begin{abstract}
We first define the concept of weighted mean method of summability and then present necessary and sufficient Tauberian conditions for the weighted mean summability of sequences in two-normed spaces. As corollaries, we establish two-normed analogues of two classical Tauberian theorems.
\end{abstract}

\section{Introduction}

The notion of two-normed spaces was first defined by Gähler [7], and has been developed extensively by several authors (see, for example, $[3,5,6,9$, $18])$.

Let $X$ be a real vector space with the $\operatorname{dimension} \operatorname{dim} X \geq 2$. A two-norm on $X$ is a function $\|\cdot, \cdot\|: X \times X \rightarrow \mathbb{R}$ which satisfies the conditions

(i) $\|x, y\|=0$ if and only if $x$ and $y$ are linearly dependent,

(ii) $\|x, y\|=\|y, x\|$ for each $x, y \in X$,

(iii) $\|\alpha x, y\|=|\alpha|\|x, y\|$ for each $x, y \in X$ and $\alpha \in \mathbb{R}$,

(iv) $\|x, y+z\| \leq\|x, y\|+\|x, z\|$ for each $x, y, z \in X$.

The pair $(X,\|\cdot, \cdot\|)$ is then called a two-normed space. Observe that in any two-normed space, $\|x, y\|$ is nonnegative and $\|x, y+\alpha x\|=\|x, y\|$ for each $x, y \in X$ and $\alpha \in \mathbb{R}$.

A standard example of a two-normed space is $\mathbb{R}^{2}$ being equipped with the two-norm

$$
\|x, y\|=\left|x_{1} y_{2}-x_{2} y_{1}\right|
$$

where $x=\left(x_{1}, x_{2}\right)$ and $y=\left(y_{1}, y_{2}\right)$.

Received March 22, 2019.

2010 Mathematics Subject Classification. 40E05, 40A05.

Key words and phrases. Tauberian conditions, weighted mean methods of summability, two-normed spaces, slowly oscillating sequences.

https://doi.org/10.12697/ACUTM.2020.24.04 
Let $\left(x_{n}: n=0,1,2, \ldots\right)$ be a sequence in a two-normed space $(X,\|\cdot, \cdot\|)$. The sequence $\left(x_{n}\right)$ is said to be convergent to $l \in X$ if for every $y \in X$,

$$
\lim _{n \rightarrow \infty}\left\|x_{n}-l, y\right\|=0 .
$$

Furthermore, $\left(x_{n}\right)$ is called bounded (notation $x_{n}=O(1)$ ) if for every $y \in X$ there exists $H>0$ such that

$$
\left\|x_{n}, y\right\| \leq H \quad(n=0,1,2, \ldots) .
$$

In 2017, Savas and Sezer [13] considered the $(C, 1)$ summability in 2normed spaces. We introduce the weighted mean method of summability in 2-normed spaces.

Let $\left(p_{n}\right)\left(n \in \mathbb{N}_{0}:=\{0,1,2, \ldots\}\right)$ be a sequence of nonnegative numbers with $p_{0}>0$ such that

$$
P_{n}:=\sum_{k=0}^{n} p_{k} \rightarrow \infty, \quad \text { as } n \rightarrow \infty .
$$

The weighted means of the sequence $\left(x_{n}\right)$ are defined by

$$
t_{n}:=\frac{1}{P_{n}} \sum_{k=0}^{n} p_{k} x_{k}, \quad n=0,1,2, \ldots
$$

The sequence $\left(x_{n}\right)$ is said to be summable to $l \in X$ by the weighted mean method determined by the sequence $\left(p_{n}\right)$ (briefly, summable $(\bar{N}, p)$ to $l \in X$ ) if for every $y \in X$,

$$
\lim _{n \rightarrow \infty}\left\|t_{n}-l, y\right\|=0 .
$$

Notice that summability method $(\bar{N}, p)$ reduces to the Cesàro method $(C, 1)$ if $p_{n}=1$ for all $n \in \mathbb{N}_{0}$, and to the logarithmic method $(\ell, 1)$ if $p_{n}=1 /(n+1)$ for all $n \in \mathbb{N}_{0}$.

The following theorem indicates that the convergence of a sequence in a two-normed space always implies the convergence of its weighted means to the same limit.

Theorem 1. Let the condition (2) be satisfied. If a sequence $\left(x_{n}\right)$ in $(X,\|\cdot, \cdot\|)$ converges to $l \in X$, then the sequence $\left(t_{n}\right)$ of its weighted means also converges to $l$.

Proof. If $\left(x_{n}\right)$ converges to $l$, then, for every $\epsilon>0$ and $y \in X$, there exists a positive integer $n_{0}$ such that $\left\|x_{n}-l, y\right\| \leq \epsilon / 2$ if $n>n_{0}$, and there exists 
$H>0$ such that $\left\|x_{n}-l, y\right\| \leq H$ if $n \leq n_{0}$. Hence, for every $y \in X$, we get

$$
\begin{aligned}
\left\|t_{n}-l, y\right\| & =\left\|\frac{1}{P_{n}} \sum_{k=0}^{n} p_{k} x_{k}-\frac{1}{P_{n}} \sum_{k=0}^{n} p_{k} l, y\right\| \\
& =\left\|\frac{1}{P_{n}} \sum_{k=0}^{n} p_{k}\left(x_{k}-l\right), y\right\| \leq \frac{1}{P_{n}} \sum_{k=0}^{n} p_{k}\left\|x_{k}-l, y\right\| \\
& =\frac{1}{P_{n}} \sum_{k=0}^{n_{0}} p_{k}\left\|x_{k}-l, y\right\|+\frac{1}{P_{n}} \sum_{k=n_{0}+1}^{n} p_{k}\left\|x_{k}-l, y\right\| \\
& \leq \frac{H P_{n_{0}}}{P_{n}}+\frac{\epsilon}{2} .
\end{aligned}
$$

Since $P_{n_{0}} / P_{n} \rightarrow 0$ as $n \rightarrow \infty$, there exists a positive integer $n_{1}$ such that $\left|H P_{n_{0}} / P_{n}\right| \leq \epsilon / 2$ if $n>n_{1}$. Therefore, $\left\|t_{n}-l, y\right\| \leq \epsilon$ if $n>\max \left\{n_{0}, n_{1}\right\}$.

Theorem 1 expresses the so-called regularity property of the weighted mean method of summability in two-normed spaces.

Example 2. Let $X=\mathbb{R}^{2}$ be equipped with the two-norm (1). Define the sequence $\left(x_{n}\right)$ in $X$ by

$$
x_{n}=\left(1+(-1)^{n+1}, \frac{1}{2}+\frac{(-1)^{n}}{2}\right),
$$

and let $y=\left(y_{1}, y_{2}\right)$. Then, putting $p_{n}=1$ for all $n$, we find the $(C, 1)$ mean of $\left(x_{n}\right)$ as

$$
t_{n}= \begin{cases}\left(\frac{n}{n+1}, \frac{n+2}{2 n+2}\right) & \text { if } n \text { is even } \\ \left(1, \frac{1}{2}\right) & \text { if } n \text { is odd }\end{cases}
$$

In the case $n$ is even, we obtain that, for all $y=\left(y_{1}, y_{2}\right) \in \mathbb{R}^{2}$,

$$
\begin{aligned}
\lim _{n \rightarrow \infty}\left\|t_{n}-l, y\right\| & =\lim _{n \rightarrow \infty}\left\|\left(\frac{n}{n+1}, \frac{n+2}{2 n+2}\right)-\left(1, \frac{1}{2}\right),\left(y_{1}, y_{2}\right)\right\| \\
& =\lim _{n \rightarrow \infty}\left\|\left(\frac{-1}{n+1}, \frac{1}{2 n+2}\right),\left(y_{1}, y_{2}\right)\right\| \\
& =\lim _{n \rightarrow \infty}\left|\frac{-y_{2}}{n+1}-\frac{y_{1}}{2 n+2}\right|=0 .
\end{aligned}
$$

If $n$ is odd, then $\left(t_{n}\right)=(1,1 / 2)$. Therefore, it follows from (3) that $\left(x_{n}\right)$ is $(C, 1)$ summable to $(1,1 / 2)$.

Besides, if $\left(x_{n}\right)$ is convergent, the limit should be $(1,1 / 2)$. However, when $y=(0,1)$, since

$$
\begin{aligned}
\lim _{n \rightarrow \infty}\left\|x_{n}-L, y\right\| & =\lim _{n \rightarrow \infty}\left\|\left((-1)^{n+1}, \frac{(-1)^{n}}{2}\right),\left(y_{1}, y_{2}\right)\right\| \\
& =\lim _{n \rightarrow \infty}\left|(-1)^{n+1} y_{2}-\frac{(-1)^{n} y_{1}}{2}\right| \\
& =\lim _{n \rightarrow \infty}\left|(-1)^{n+1}\right|=1 \neq 0
\end{aligned}
$$


we have that the sequence $\left(x_{n}\right)$ is not convergent.

Example 2 shows that the converse of Theorem 1 is not true in general. Thus we are led to the problem of finding additional conditions which, together with assumption (3), would assure the convergence of the sequence $\left(x_{n}\right)$. Such conditions are called Tauberian conditions and the resulting theorem is called a Tauberian theorem. Tauberian type theorems have a long history: see, for example, the book [10] and the papers $[1,2,4,12,16,17]$.

In this work, our purpose is to obtain Tauberian conditions under which the convergence of $\left(x_{n}\right)$ in a two-normed space follows from its $(\bar{N}, p)$ summability.

\section{Main results}

Theorem 3. Let $\left(p_{n}\right)$ be a sequence of nonnegative numbers such that $p_{0}>0$ and

$$
\liminf _{n \rightarrow \infty} \frac{P_{[\lambda n]}}{P_{n}}>1 \text { for every } \lambda>1,
$$

where $[\lambda n]$ denotes the integer part of the product $\lambda n$, and let $\left(x_{n}\right)$ be a sequence in $(X,\|\cdot, \cdot\|)$ which is $(\bar{N}, p)$ summable to $l \in X$. Then $\left(x_{n}\right)$ converges to $l$ if and only if one of the following two conditions is satisfied:

$$
\begin{aligned}
& \inf _{\lambda>1} \limsup _{n \rightarrow \infty}\left\|\frac{1}{P_{[\lambda n]}-P_{n}} \sum_{k=n+1}^{[\lambda n]} p_{k}\left(x_{k}-x_{n}\right), y\right\|=0, \\
& \inf _{0<\lambda<1} \limsup _{n \rightarrow \infty}\left\|\frac{1}{P_{n}-P_{[\lambda n]}} \sum_{k=[\lambda n]+1}^{n} p_{k}\left(x_{n}-x_{k}\right), y\right\|=0
\end{aligned}
$$

for all $y \in X$.

Remark 4. It is clear that the condition (4) implies (2).

Remark 5. Consider the special cases of (5) and (6) for $p_{n}=1$ for all $n \in \mathbb{N}_{0}$. In this case, conditions (5) and (6) reduce to

$$
\inf _{\lambda>1} \limsup _{n \rightarrow \infty}\left\|\frac{1}{[\lambda n]-n} \sum_{k=n+1}^{[\lambda n]}\left(x_{k}-x_{n}\right), y\right\|=0
$$

and

$$
\inf _{0<\lambda<1} \limsup _{n \rightarrow \infty}\left\|\frac{1}{n-[\lambda n]} \sum_{k=[\lambda n]+1}^{n}\left(x_{n}-x_{k}\right), y\right\|=0,
$$

respectively.

Savaş and Sezer [13] used Tauberian conditions (7) and (8) to retrieve convergence of $\left(x_{n}\right)$ from the $(C, 1)$ summability. 
Remark 6. Following Schmidt [14] (or Stanojević [15]), a sequence $\left(x_{n}\right)$ is said to be slowly oscillating in two-norm if for every $y \in X$,

$$
\inf _{\lambda>1} \limsup _{n \rightarrow \infty} \max _{n<k \leq[\lambda n]}\left\|x_{k}-x_{n}, y\right\|=0
$$

or equivalently,

$$
\inf _{0<\lambda<1} \limsup _{n \rightarrow \infty} \max _{[\lambda n]<k \leq n}\left\|x_{n}-x_{k}, y\right\|=0 .
$$

Notice that, if $\left(x_{n}\right)$ is slowly oscillating in two-norm, then (7) and (8) hold.

Remark 7. If the two-sided condition $\frac{P_{n}}{p_{n}} \Delta x_{n}=O(1)$ of Hardy [8] type, where $\Delta x_{n}=x_{n}-x_{n-1}$, is satisfied under some appropriate condition imposed on $\left(p_{n}\right)$, then $\left(x_{n}\right)$ is slowly oscillating in two-norm.

Taking into account the Remarks 6 and 7, we obtain the following twonormed analogues of some classical Tauberian theorems.

Corollary 8. Let (4) be satisfied and let $\left(x_{n}\right)$ be $(\bar{N}, p)$ summable to $l \in X$. If $\left(x_{n}\right)$ is slowly oscillating in two-norm, then $\left(x_{n}\right)$ converges to $l$.

Corollary 9. Let

$$
\lim _{n \rightarrow \infty} \frac{P_{[\lambda n]}}{P_{n}}=\lambda^{\delta}, \quad \lambda>1,
$$

be satisfied for some $\delta>0$ and let $\left(x_{n}\right)$ be $(\bar{N}, p)$ summable to $l \in X$. If

$$
\frac{P_{n}}{p_{n}} \Delta x_{n}=O(1)
$$

then $\left(x_{n}\right)$ converges to $l$.

Remark 10. It is clear that the condition (10) implies (4).

\section{Auxiliary results}

Lemma 11 (see [11]). If $\left(P_{n}\right)$ is a nondecreasing sequence of positive numbers, then (4) is equivalent to the condition

$$
\liminf _{n \rightarrow \infty} \frac{P_{n}}{P_{[\lambda n]}}>1 \quad \text { for every } 0<\lambda<1 .
$$

In the proof of the main theorem we need the following lemma on the so-called moving weighted averages.

Lemma 12. Let $\left(p_{n}\right)$ be a sequence of nonnegative real numbers such that $p_{0}>0$ and the condition (4) is satisfied. If $\left(x_{n}\right)$ is $(\bar{N}, p)$ summable to $l \in X$, then, for each $y \in X$,

$$
\lim _{n \rightarrow \infty}\left\|\frac{1}{P_{[\lambda n]}-P_{n}} \sum_{k=n+1}^{[\lambda n]} p_{k} x_{k}-l, y\right\|=0 \quad \text { for every } \lambda>1
$$


and

$$
\lim _{n \rightarrow \infty}\left\|\frac{1}{P_{n}-P_{[\lambda n]}} \sum_{k=[\lambda n]+1}^{n} p_{k} x_{k}-l, y\right\|=0 \quad \text { for every } \quad 0<\lambda<1 .
$$

Proof. For brevity, we denote the moving weighted averages $\left(\tau_{n}^{>}\right)$for $\lambda>1$ and $\left(\tau_{n}^{<}\right)$for $0<\lambda<1$, respectively, by

$$
\tau_{n}^{>}=\frac{1}{P_{[\lambda n]}-P_{n}} \sum_{k=n+1}^{[\lambda n]} p_{k} x_{k} \text { and } \tau_{n}^{<}=\frac{1}{P_{n}-P_{[\lambda n]}} \sum_{k=[\lambda n]+1}^{n} p_{k} x_{k} .
$$

Consider the case $\lambda>1$. Then

$$
\begin{aligned}
& \left\|\tau_{n}^{>}-l, y\right\|=\left\|\tau_{n}^{>}+t_{[\lambda n]}-t_{[\lambda n]}-l, y\right\|=\| \frac{1}{P_{[\lambda n]}-P_{n}} \sum_{k=0}^{[\lambda n]} p_{k} x_{k} \\
& \quad-\frac{1}{P_{[\lambda n]}-P_{n}} \sum_{k=0}^{n} p_{k} x_{k}+\frac{1}{P_{[\lambda n]}} \sum_{k=0}^{[\lambda n]} p_{k} x_{k}-\frac{1}{P_{[\lambda n]}} \sum_{k=0}^{[\lambda n]} p_{k} x_{k}-l, y \| \\
& =\left\|\frac{P_{n}}{P_{[\lambda n]}-P_{n}} \frac{1}{P_{[\lambda n]}} \sum_{k=0}^{[\lambda n]} p_{k} x_{k}-\frac{P_{n}}{P_{[\lambda n]}-P_{n}} \frac{1}{P_{n}} \sum_{k=0}^{n} p_{k} x_{k}+\frac{1}{P_{[\lambda n]}} \sum_{k=0}^{[\lambda n]} p_{k} x_{k}-l, y\right\| .
\end{aligned}
$$

Thus we see that

$$
\left\|\tau_{n}^{>}-l, y\right\| \leq \frac{P_{n}}{P_{[\lambda n]}-P_{n}}\left\|t_{[\lambda n]}-t_{n}, y\right\|+\left\|t_{[\lambda n]}-l, y\right\| .
$$

Moreover, from (4) we have

$$
\limsup _{n \rightarrow \infty} \frac{P_{n}}{P_{[\lambda n]}-P_{n}}=\left(\liminf _{n \rightarrow \infty} \frac{P_{[\lambda n]}}{P_{n}}-1\right)^{-1}<\infty .
$$

Now, (13) follows from (15) and the $(\bar{N}, p)$ summability of $\left(x_{n}\right)$ to $l$. The proof of (14) is similar to that of (13).

\section{Proofs of main results}

Proof of Theorem 3. Necessity. Assume that $\left(x_{n}\right)$ is convergent to $l$. Given any $\lambda>1$, by Lemma 12 we obtain

$$
\begin{aligned}
& \lim _{n \rightarrow \infty}\left\|\frac{1}{P_{[\lambda n]}-P_{n}} \sum_{k=n+1}^{[\lambda n]} p_{k}\left(x_{k}-x_{n}\right), y\right\| \\
& \leq \lim _{n \rightarrow \infty}\left\|\frac{1}{P_{[\lambda n]}-P_{n}} \sum_{k=n+1}^{[\lambda n]} p_{k} x_{k}-l, y\right\|+\lim _{n \rightarrow \infty}\left\|x_{n}-l, y\right\|=0
\end{aligned}
$$


for every $y \in X$. Namely, we have an even stronger condition than (5). In a similar way, for any $0<\lambda<1$, we have

$$
\lim _{n \rightarrow \infty}\left\|\frac{1}{P_{n}-P_{[\lambda n]}} \sum_{k=[\lambda n]+1}^{n} p_{k}\left(x_{n}-x_{k}\right), y\right\|=0,
$$

which is stronger than (6).

Sufficiency. Suppose that (5) holds, and let $y \in X$ be arbitrarily fixed. Then, for any given $\epsilon>0$, there exists $\lambda>0$ such that

$$
\limsup _{n \rightarrow \infty}\left\|\frac{1}{P_{[\lambda n]}-P_{n}} \sum_{k=n+1}^{[\lambda n]} p_{k}\left(x_{k}-x_{n}\right), y\right\| \leq \epsilon .
$$

So, using also $(\bar{N}, p)$ summability of $\left(x_{n}\right)$ and Lemma 12 , we obtain

$$
\begin{aligned}
\limsup _{n \rightarrow \infty}\left\|x_{n}-l, y\right\| \leq & \limsup _{n \rightarrow \infty}\left\|\frac{1}{P_{[\lambda n]}-P_{n}} \sum_{k=n+1}^{[\lambda n]} p_{k} x_{k}-l, y\right\| \\
& +\limsup _{n \rightarrow \infty}\left\|\frac{1}{P_{[\lambda n]}-P_{n}} \sum_{k=n+1}^{[\lambda n]} p_{k}\left(x_{k}-x_{n}\right), y\right\| \leq \epsilon
\end{aligned}
$$

for every $y \in X$. Since $\epsilon>0$ is arbitrary, convergence of $\left(x_{n}\right)$ to $l$ follows.

A similar proof can be given if (6) is satisfied.

Proof of Corollary 8. Suppose that $\left(x_{n}\right)$ is slowly oscillating in two-norm. Then, for any given $y \in X$, we have

$$
\begin{aligned}
\left\|\frac{1}{P_{[\lambda n]}-P_{n}} \sum_{k=n+1}^{[\lambda n]} p_{k}\left(x_{k}-x_{n}\right), y\right\| & \leq \frac{1}{P_{[\lambda n]}-P_{n}} \sum_{k=n+1}^{[\lambda n]} p_{k}\left\|x_{k}-x_{n}, y\right\| \\
& \leq \max _{n<k \leq[\lambda n]}\left\|x_{k}-x_{n}, y\right\| .
\end{aligned}
$$

Taking the limsup of the last inequality as $n \rightarrow \infty$, we get

$$
\limsup _{n \rightarrow \infty}\left\|\frac{1}{P_{[\lambda n]}-P_{n}} \sum_{k=n+1}^{[\lambda n]} p_{k}\left(x_{k}-x_{n}\right), y\right\| \leq \limsup _{n \rightarrow \infty} \max _{n<k \leq[\lambda n]}\left\|x_{k}-x_{n}, y\right\| .
$$

Therefore, we conclude that

$$
\inf _{\lambda>1} \limsup _{n \rightarrow \infty}\left\|\frac{1}{P_{[\lambda n]}-P_{n}} \sum_{k=n+1}^{[\lambda n]} p_{k}\left(x_{k}-x_{n}\right), y\right\|=0
$$

for every $y \in X$. The proof follows from Theorem 3 . 
Proof of Corollary 9. Let (10) and (11) be satisfied. Then, for every $y \in X$, there exists $H>0$ such that $\left\|\frac{P_{n}}{p_{n}} \Delta x_{n}, y\right\| \leq H \quad(n=0,1, \ldots)$. Hence, slow oscillation of $\left(x_{n}\right)$ follows. Indeed, for each $y \in X$,

$$
\begin{aligned}
\left\|x_{k}-x_{n}, y\right\| & =\left\|\sum_{j=n+1}^{k}\left(x_{j}-x_{j-1}\right), y\right\|=\left\|\sum_{j=n+1}^{k} \Delta x_{j}, y\right\| \\
& \leq \sum_{j=n+1}^{k} \frac{p_{j}}{P_{j}}\left\|\Delta x_{j}, y\right\| \leq H \sum_{j=n+1}^{k} \frac{p_{j}}{P_{j}} \\
& \leq H \frac{P_{k}-P_{n}}{P_{n}} .
\end{aligned}
$$

Taking the maximum of both sides of the inequality above, we have

$$
\max _{n<k \leq[\lambda n]}\left\|x_{k}-x_{n}, y\right\| \leq H \frac{P_{[\lambda n]}-P_{n}}{P_{n}} .
$$

Now, taking the limsup of both sides of the last inequality, by (10) we have that

$$
\limsup _{n \rightarrow \infty} \max _{n<k \leq[\lambda n]}\left\|x_{k}-x_{n}, y\right\| \leq H\left(\lambda^{\delta}-1\right) .
$$

Finally, taking the infimum of both sides of (16) for $\lambda>1$, we get

$$
\inf _{\lambda>1} \limsup _{n \rightarrow \infty} \max _{n<k \leq[\lambda n]}\left\|x_{k}-x_{n}, y\right\|=0 .
$$

Thus, the proof is completed by Corollary 8 .

\section{Acknowledgment}

The authors would like to thank the anonymous referee for many constructive comments and helpful corrections.

\section{References}

[1] İ. Çanak, Z. Önder, and Ü. Totur, Statistical extensions of some classical Tauberian theorems for Cesàro summability of triple sequences, Results Math. 70 (2016), 457473.

[2] M. Dik, Tauberian theorems for sequences with moderately oscillatory control modulo, Math. Morav. 5 (2001), 57-94.

[3] C. Diminnie, S. Gähler, and A. White, 2-inner product spaces, Demonstratio Math. 6 (1973), 525-536.

[4] Y. Erdem, Tauberian conditions on some slowly decreasing sequences, Period. Math. Hung. 78 (2019), 38-46.

[5] R. W. Freese and Y. J. Cho, Geometry of Linear 2-Normed Spaces, Nova Science Publishers, New York, 2001.

[6] R. W. Freese, Y. J. Cho, and S. S. Kim, Strictly 2-convex linear 2-normed spaces, J. Korean Math. Soc. 29 (1992), 391-400.

[7] S. Gähler, Lineare 2-normierte Räume, Math. Nachr. 28 (1964), 1-43. 
[8] G. H. Hardy, Theorems relating to the summability and convergence of slowly oscillating series, Proc. London Math. Soc. 8 (1910), 301-320.

[9] S. S. Kim, Y. J. Cho, and A. White, Linear operators on linear 2-normed spaces, Glasnik Mat. 27 (1992), 63-70.

[10] J. Korevaar, Tauberian Theory: A Century of Developments, Springer-Verlag, Berlin, 2004.

[11] F. Móricz and B. E. Rhoades, Necessary and sufficient Tauberian conditions for certain weighted mean methods of summability. II, Acta Math. Hungar. 102 (2004), 279-285.

[12] M. A. Okur and Ü. Totur, Tauberian theorems for the logarithmic summability methods of integrals, Positivity. 23 (2019), 55-73.

[13] R. Savaş and S. A. Sezer, Tauberian theorems for sequences in 2-normed spaces, Results Math. 72 (2017), 1919-1931.

[14] R. Schmidt, Über divergente Folgen und lineare Mittelbildungen, Math. Z. 22 (1925), 89-152.

[15] Č. V. Stanojević, Slow oscillation in norm and structure of linear functionals, Publ. Inst. Math. (Beograd) (N.S.) 58(72) (1995), 93-100.

[16] Ü. Totur and İ. Çanak, Tauberian conditions for the $(C, \alpha)$ integrability of functions, Positivity 21 (2017), 73-83.

[17] Ü. Totur, I. Çanak, and S. A. Sezer, Weighted integrability and its applications in quantum calculus, Proc. Natl. Acad. Sci. India Sect. A Phys. Sci. 89 (2019), 791-797.

[18] A. G. White Jr., 2-Banach spaces, Math. Nachr. 42 (1969), 43-60.

Department of Mathematics, Ege University, İzmir 35100, Turkey

E-mail address: ibrahim.canak@ege.edu.tr

E-mail address: 91180000453@ogrenci.ege.edu.tr

Department of Mathematics İstanbul Medeniyet University, Istanbul 34720 , TURKEY

E-mail address: sefaanil.sezer@medeniyet.edu.tr

Department of Mathematics, Ege University, İzmir 35100, Turkey

E-mail address: yarasgilece@gmail.com 